(2) OPEN ACCESS

\title{
Normative data and percentile curves for axial length and axial length/corneal curvature in Chinese children and adolescents aged $4-18$ years
}

\author{
Xiangui He (ㄷ) 1,2,3 Padmaja Sankaridurg 주, 4,5 Thomas Naduvilath, 4,5 \\ Jingjing Wang (1) ${ }^{1,2,3}$ Shuyu Xiong, ${ }^{1,2,3}$ Rebecca Weng, ${ }^{4}$ Linlin Du, ${ }^{1,2,3}$ Jun Chen, ${ }^{1,2,3}$ \\ Haidong Zou, ${ }^{1,2,3}$ Xun Xu (1) ${ }^{1,2,3}$
}

\begin{abstract}
'Shanghai Eye Disease Prevention and Treatment Center, Shanghai Eye Hospital, Shanghai Vision Health Center \& Shanghai Children Myopia Institute, Shanghai, China ${ }^{2}$ Department of Ophthalmology, Shanghai General Hospital, Shanghai Jiao Tong University, Center of Eye Shanghai Key Laboratory of Ocular Fundus Disease, Shanghai, China ${ }^{3}$ National Clinical Research Center for Eye Diseases, Shanghai, China

${ }^{4}$ Brien Holden Vision Institute, Sydney, New South Wales, Australia

${ }^{5}$ School of Optometry and Vision Science, University of New South Wales, Sydney, New South Wales, Australia
\end{abstract}

\section{Correspondence to} Padmaja Sankaridurg, Brien Holden Vision Institute, Sydney 2052, New South Wales, Australia;

p.sankaridurg@bhvi.org Dr Xun Xu;

drxuxun@sjtu.edu.cn

Received 9 April 2021 Accepted 1 September 2021

Check for updates

(C) Author(s) (or their employer(s)) 2021. Re-use permitted under CC BY-NC. No commercial re-use. See rights and permissions. Published by BMJ.

To cite: He X, Sankaridurg $P_{\text {, }}$ Naduvilath T, et al.

Br J Ophthalmol Epub ahead of print: [please include Day Month Year]. doi:10.1136/ bjophthalmol-2021-319431

\begin{abstract}
Purpose To develop age-specific and gender-specific reference percentile charts for axial length $(A L)$ and $\mathrm{AL} /$ corneal radius of curvature $(\mathrm{AL} / \mathrm{CR})$ and, to use percentiles to determine probability of myopia and estimate refractive error (RE).

Methods Analysis of $A L$, cycloplegic RE and $C R$ of 14127 Chinese participants aged 4-18years from 3 studies. AL and AL/CR percentiles estimated using Lambda-Mu-Sigma method and compared for agreement using intraclass correlation (ICC). Logistic regression was used to model risk of myopia based on age, gender, $A L$ and $A L / C R$ percentiles. Accuracy of AL progression and RE estimated using percentiles was validated using an independent sample of 5742 eyes of children aged 7-10years.
\end{abstract}

Results Age-specific and gender-specific AL and AL/ CR (3rd, 5th, 10th, 25th, 50th, 75th, 90th and 95th) percentiles are presented. Concordance between $A L$ and $\mathrm{AL} / \mathrm{CR}$ percentiles improved with age $(0.13$ at 4 years to $>0.75$ from 13 years) and a year-to-year change was observed for all except $<10$ th percentile from 15 years. Increasing age, $\mathrm{AL}$ and $\mathrm{AL} / \mathrm{CR}$ was associated with a more myopic $R E\left(r^{2}=0.45,0.70\right.$ and 0.83 , respectively). The sensitivity and specificity of the model to estimate probability of myopia was $86.0 \%$ and $84.5 \%$, respectively. Estimation of 1-year change in AL using percentiles correlated highly with actual $\mathrm{AL}(\mathrm{ICC}=0.98)$. Concordance of estimated to actual RE was high (ICC $=0.80$ ) and within $\pm 0.50 \mathrm{D}$ and $\pm 1.0 \mathrm{D}$ of actual RE for $47.4 \%$ and $78.9 \%$ of eyes, respectively.

Conclusion Age-specific and gender-specific $A L$ and $A L /$ CR percentiles provide reference data, aid in identifying and monitoring individuals at risk of myopia and have utility in screening for myopia. AL/CR percentiles were more accurate in estimating probability of myopia in younger children.

\section{INTRODUCTION}

Myopia or short-sightedness is the most common cause of distance vision impairment and is estimated to affect nearly half of the world's population by the year $2050 .^{1}$ The presence of any myopia indicates that the eye length has extended beyond 'the eye length resulting in emmetropia' causing a mismatch between the optical components of the eye, such as the cornea and the eye length and therefore, resulting in blurred distance vision. Although vision is easily restored with corrective strategies such as spectacles or contact lenses, the axial elongation often continues well into teenage years and in few, into adulthood leading to further progression of myopia. Any level of myopia imposes an economic and health burden, ${ }^{2}$ and importantly, progression to higher levels may lead to uncorrectable vision impairment as well as sight-threatening complications such as myopic macular degeneration. ${ }^{34}$ Although myopia is detected commonly in schoolaged children, evidence indicates that myopia is linked to education. ${ }^{5}$ For example, an early onset of myopia is observed in regions where schooling commences early, ${ }^{67}$ and is also detected in high school and university graduates coinciding with periods of academic demand. ${ }^{8}{ }^{9}$ An early onset of myopia is likely to result in more number of years in the progression mode and therefore resulting in a higher net myopia. ${ }^{10}$

Although, there are several risk factors associated with myopia such as age, parental myopia, education, urban living environment with reduced time outdoors, etc, ${ }^{511}$ the aforementioned risk factors alone have not been sufficient to accurately determine the risk of onset and progression. A refractive error assessment is commonly used to categorise an eye as myopic and monitor progression, and a low hyperopic refractive error in young children is considered predictive of future myopia. ${ }^{12}$ However, when the refractive error assessment does not involve cycloplegia, errors due to overaccommodation are common. ${ }^{13}$ Also, the accuracy and repeatability of refractive error measurements including subjective refraction are within $\pm 0.50 \mathrm{D} .{ }^{14-16}$ In this regard, modern technology for axial length (AL) measurements using partial coherence interferometry and swept-source techniques is more accurate; measurement errors and variability between instruments are limited to few microns. ${ }^{17} 18$ Additionally, techniques to measure AL are rapid, noninvasive and can be easy for both the practitioner and the young child. Thus, utilising AL measures either alone or in combination with other ocular measurements (eg, corneal curvature) to differentiate normal from excessive ocular growth provides clinicians with a powerful tool to identify at risk children. Indeed, it has been reported that although $\mathrm{AL}$ is the main determinant of excessive eye length underlying myopia, $\mathrm{AL} /$ corneal radius of curvature $(\mathrm{AL} / \mathrm{CR})$ is a more robust measure of the refractive 
Table 1 Details of study cohort (development data set)

\begin{tabular}{|c|c|c|c|c|}
\hline \multirow[b]{2}{*}{ Participant details } & \multicolumn{3}{|c|}{ Development data set } & \multirow[b]{2}{*}{ Validation cohort $^{21}$} \\
\hline & Study cohort $1^{13}$ & Study cohort $2^{21}$ & Study cohort 3 (unpublished) & \\
\hline No of participants & 6017 & 6293 & 1817 & 5742 \\
\hline Age (years) & $9.1 \pm 2.9$ (4.0 to 16.0$)$ & $7.2 \pm 0.6$ (6.1 to 9.4$)$ & $18.2 \pm 0.3(17.9$ to 19.0$)$ & $8.4 \pm 0.6$ (7.1 to 10.4$)$ \\
\hline Male: female $\%$ & 54.3: 45.7 & $53.2: 46.8$ & $58.0: 42.0$ & 52.8: 47.2 \\
\hline Axial length (mm) & $23.4 \pm 1.2(18.3$ to 29.4$)$ & $22.9 \pm 0.8(19.5$ to 25.9$)$ & $25.1 \pm 1.3$ (20.9 to 30.4$)$ & $23.2 \pm 0.8(20.0$ to 26.4$)$ \\
\hline $\mathrm{AL} / \mathrm{CR}$ ratio & $3.0 \pm 0.1$ (2.5 to 3.9 ) & $2.9 \pm 0.1$ ( 2.5 to 3.5$)$ & $3.2 \pm 0.2(2.7$ to 3.8$)$ & $3.0 \pm 0.1$ (2.5 to 3.5$)$ \\
\hline $\operatorname{SE}(D)$ & $-0.1 \pm 2.0(-10.8$ to 9.0$)$ & $1.0 \pm 1.0(-8.9$ to 8.9$)$ & $-3.6 \pm 2.6(-13.1$ to 6.9$)$ & $0.5 \pm 1.2(-9.0$ to 8.4$)$ \\
\hline
\end{tabular}

$A L$, axial length; $C R$, corneal radius; $S E$, spherical equivalent .

status of the eye; the AL/CR ratio correlates more strongly with the spherical equivalent (SE) refractive error compared with AL alone. ${ }^{19}{ }^{20}$ Furthermore, as an increase in myopia is primarily due to axial elongation, estimating and monitoring AL change is advantageous.

Therefore, we sought to develop and validate AL and AL/CR percentile curves from a large data set of measurements obtained from Chinese children. The purpose was to (1) develop percentile charts for $\mathrm{AL}$ and $\mathrm{AL} / \mathrm{CR}$ with consideration to the influence of age and gender and (2) to determine if the percentile charts can be used to determine the probability of myopia and estimate refractive error.

\section{METHODS}

\section{Patient population}

A retrospective analysis of participant data on AL, cycloplegic SE and corneal curvature collected from 14127 participants aged 4-18 years from baseline data of three individual studies (referred to as development dataset, table 1). Details of the design and methodology of two of the three studies were previously published. ${ }^{1321}$ The third study included data from 1817 young adults aged mostly 17-18 years from Shanghai region. To validate the data, the follow-up data (12-24 months) of 5742 children (7-10years) of 1 of the 3 studies from development dataset was considered (table 1$).^{21}$

\section{Data}

Prior to enrolment in respective studies, informed consent for each participant was obtained from parent/carer or the participant (in the case of the young adult). The data sets of all studies did not include data of participants with any systemic or ocular pathology, strabismus or amblyopia or those that had used any myopia control treatments.

Each participant from the development dataset had undergone an eye examination that included an objective cycloplegic refraction (two drops of $1 \%$ cyclopentolate eye-drops preceded by $0.5 \%$ proparacaine eye-drops) with refractive error measured with an autorefractor (KR-8900, Topcon, Tokyo, Japan). Eye length was measured using an IOL Master (V.5.02, Carl Zeiss, Jena, Germany). Only the baseline measurements were considered for the analysis. The corneal curvature data were obtained from the IOL Master and included the radii of curvature of steep and flat meridians.

Similarly, the validation dataset included a cycloplegic refractive error assessment collected using autorefractor (KR8900, Topcon, Tokyo, Japan) and an AL and corneal curvature measurement using IOL Master (version 5.02, Carl Zeiss, Jena, Germany). Cycloplegia was obtained using two drops of $1 \%$ cyclopentolate eye-drops.

\section{Statistical analysis}

The development data set was skewed to younger ages and therefore age specific sample weights were used to standardise the sample to the estimated population distribution for ages $4-18$ years. $^{22}$ Data of both eyes were considered. AL/CR ratio was computed as a ratio of the AL $(\mathrm{mm})$ by the average of the radii of curvature of the flat and steep meridians of the cornea (mm). SE was computed as sphere plus half cylinder in dioptres. Myopia was defined as SE $\leq-0.50 \mathrm{D}$. The baseline ocular characteristics, that is, $\mathrm{AL}$, corneal curvature, $\mathrm{AL} / \mathrm{CR}$ and $\mathrm{SE}$ of the study population was summarised by age and gender as mean \pm SD and with boxplots.

Percentile curves based on age were developed using the LMS (lambda-mu-sigma) method, ${ }^{23}$ which summarises the changing distribution of ocular parameters across age by estimating three parameters as a function of age. These three parameters are L (lambda, skewness) expressed as a Box-Cox power, M (mu, median) and S (sigma, coefficient of variation). Penalised likelihood was used to curve fit as cubic splines the three parameters as a function of age using non-linear regression. Smoothening was achieved by adjusting the equivalent df. A model that minimised deviance restricting the $\mathrm{df}$ to a maximum of 5 was chosen as the final model and the resulting values of $\mathrm{L}, \mathrm{M}$ and $\mathrm{S}$ as a function of age was exported to plot percentile curves. The nth percentile of an ocular parameter such as AL at an age $\mathrm{y}$ was then computed as:

$\mathrm{M}_{\mathrm{y}} \times\left(1+\left(\mathrm{L}_{\mathrm{y}} \times \mathrm{S}_{\mathrm{y}} \times \mathrm{Z}_{\mathrm{n}}\right)^{\wedge}\left(1 / \mathrm{L}_{\mathrm{y}}\right)\right.$

Where $Z_{n}$ is the standard normal variate at the $n^{\text {th }}$ percentile with mean of 0 and SD of 1 and $\mathrm{L}_{\mathrm{y}}, \mathrm{M}_{\mathrm{y}}$, and $\mathrm{S}_{\mathrm{y}}$ are the parameters at age $y$.

$\mathrm{AL}$ and $\mathrm{AL} / \mathrm{CR}$ percentiles were compared for agreement at each age group using intraclass correlation (ICC).

$\mathrm{AL}$ and $\mathrm{AL} / \mathrm{CR}$ percentiles along with age and gender were used in a binary logistic model and the model equation was used to predict the individual probability of myopia and therefore myopia prevalence for specific ages and for both genders. Log likelihood and area under ROC (receiver operating characteristic) curve (AUROC) were used to summarise fit and predictability of the model.

Additionally, the weighted study sample was used to develop a model to estimate SE. Non-linear regression that included main effects, interaction and quadratic terms was used to develop the model and included age, gender, AL and AL/CR, a quadratic term of age and the interaction terms of age and gender with AL/CR.(32.5 $+(1.1 *$ Age $)+(-0.52 * \mathrm{AL})+(-7.26 * \mathrm{AL} / \mathrm{CR})+-0.30 * \mathrm{Age} * \mathrm{AL}-$ $\mathrm{CR})+\left(-0.008 * \mathrm{Age}^{2}\right)+(1.2 * \mathrm{AL} / \mathrm{CR})-3.13$ for males and 32.5 $+(1.1 *$ Age $)+(-0.52 * \mathrm{AL})+(-7.26 * \mathrm{AL} / \mathrm{CR})+(-0.30 * \mathrm{Age} * \mathrm{AL} /$ $\mathrm{CR})+\left(-0.008 * \mathrm{Age}^{2}\right)$ for females $)$. The SE of the model was used to determine the prediction interval, given as $1.96 \times \mathrm{SE}$ of the regression estimate. 


\begin{tabular}{lcccc}
\hline \multirow{4}{*}{ Table 2 } & Age gender distribution \\
\cline { 2 - 5 } $\begin{array}{l}\text { Age } \\
\text { (years) }\end{array}$ & Males & Females & Total \\
\hline 4 & $240(3.1)$ & $185(2.9)$ & $425(3.0)$ & 8.5 \\
\hline 5 & $242(3.2)$ & $205(3.2)$ & $447(3.2)$ & 8.2 \\
6 & $1601(20.9)$ & $1472(22.8)$ & $3073(21.8)$ & 7.9 \\
7 & $2091(27.3)$ & $1783(27.6)$ & $3874(27.4)$ & 7.7 \\
8 & $892(11.6)$ & $703(10.9)$ & $1595(11.3)$ & 7.5 \\
9 & $424(5.5)$ & $356(5.5)$ & $780(5.5)$ & 7.4 \\
10 & $384(5.0)$ & $319(4.9)$ & $703(5.0)$ & 7.1 \\
11 & $251(3.3)$ & $215(3.3)$ & $466(3.3)$ & 7.0 \\
12 & $123(1.6)$ & $106(1.6)$ & $229(1.6)$ & 6.9 \\
13 & $163(2.1)$ & $151(2.3)$ & $314(2.2)$ & 6.9 \\
14 & $112(1.5)$ & $77(1.2)$ & $189(1.3)$ & 6.7 \\
15 & $93(1.2)$ & $122(1.9)$ & $215(1.5)$ & 6.8 \\
17 & $464(6.1)$ & $414(6.4)$ & $878(6.2)$ & 5.8 \\
\hline 18 & $589(7.7)$ & $350(5.4)$ & $939(6.6)$ & 5.5 \\
All ages & $7669(100.0)$ & $6458(100.0)$ & $14127(100.0)$ & 100.0 \\
\hline
\end{tabular}

The percentiles and SE model were validated using the validation data set. For the analysis, the 12-month and 24-month visits of the validation set were considered as baseline and 1-year follow-up visit, respectively. Age-specific and gender-specific $\mathrm{AL}$ and $\mathrm{AL} / \mathrm{CR}$ percentiles were estimated for each participant of the validation data set. These percentiles were then used to predict the $\mathrm{AL}$ and $\mathrm{AL} / \mathrm{CR}$ at the 1-year follow -up visit. The predicted $\mathrm{AL}$ and $\mathrm{AL} / \mathrm{CR}$ were then compared with the actual $\mathrm{AL}$ ad $\mathrm{AL} / \mathrm{CR}$ at the 1-year follow-up visit using ICC and the difference between predicted and actual computed and categorised into groups: $\pm 0.15 \mathrm{~mm}, \pm 0.2 \mathrm{~mm}$ etc. The equation of the SE model was applied to the 1-year follow-up of the validation sample using percentile-based predictions of $\mathrm{AL}$ and $\mathrm{AL} /$ CR to further predict SE for each eye. The difference between the estimated and actual SE was computed and categorised into groups: $\pm 0.5 \mathrm{D}, \pm 1 \mathrm{D}$, etc. The correlation of estimated and the actual SE was computed with an accuracy level of $\geq 75 \%$ considered acceptable.

\section{RESULTS}

\section{Demographic data}

The agewise distribution of the 14127 participants is presented in table 1. The average refractive error was myopic, with a weighted mean average SE of $-0.76 \pm 2.53 \mathrm{D}(+9.00$ to $-13.00 \mathrm{D}), \mathrm{AL}$ of $23.7 \pm 1.4 \mathrm{~mm} \quad(18.3-30.4 \mathrm{~mm})$, corneal curvature of $7.85 \pm 0.25 \mathrm{~mm}(7.05-9.48 \mathrm{~mm})$ and $\mathrm{AL} / \mathrm{CR}$ ratio of $3.03 \pm 0.17$ (2.49-3.88). As expected, with increasing age, there was an increase in AL and a myopic shift in refractive error, whereas the corneal curvature was found to be relatively stable with little variation between ages (table 2 , figure 1 ). The $\mathrm{AL} / \mathrm{CR}$ values also increased with age. The population was on an average myopic at age 10 and myopia prevalence increased substantially thereafter.

There were gender differences (table 3 ) with females having a shorter AL, a steeper corneal curvature, a slightly lower AL/CR ratio and a more myopic refractive error $(\mathrm{p}<0.001)$. Figure 2 presents the weighted mean $\mathrm{AL}$ and $\mathrm{AL} / \mathrm{CR}$ and shows that for a given $\mathrm{AL}$ and $\mathrm{AL} / \mathrm{CR}$ value, females had a more myopic refractive error. For example, in females vs males, at an AL of $24 \mathrm{mms}$, the $\mathrm{SE}$ was $-1.47 \pm 1.48 \mathrm{D}$ vs $-0.26 \pm 1.24 \mathrm{D}$ and for an $\mathrm{AL} / \mathrm{CR}$ value of 3.0, the mean $S E$ was $-0.13 \pm 0.97 \mathrm{D}$ vs $0.15 \pm 0.89 \mathrm{D}$, respectively. As observed from figure 2, the differences were more apparent from the onset of myopia.

The validation sample comprised 5742 eyes of 5742 participants aged $8.4 \pm 0.6$ years ( $7-10$ years) a mean SE of $0.54 \pm 1.24 \mathrm{D}$ (+8.38 to $-9.00 \mathrm{D})$ and mean AL of $23.2 \pm 0.82 \mathrm{~mm}$ (19.97$26.37 \mathrm{~mm}$ ). The prevalence of myopia in the validation data set was slightly less than the prevalence observed in development data set for similar ages ( $16 \%$ vs $19.7 \%$ for validation sample vs development data set respectively, $\mathrm{p}<0.05$ ).

\section{Percentile curves}

Tables 4 and 5 (figure 3) provide AL and AL/CR age and gender specific 3rd, 5th, 10th, 25th, 50th, 75th, 90th and 95th percentiles. Both $\mathrm{AL}$ and $\mathrm{AL} / \mathrm{CR}$ percentiles demonstrate that for a given age, the distribution or spread of $\mathrm{AL}$ and $\mathrm{AL} / \mathrm{CR}$ was narrow at younger ages and increased with age. For example, at 4 years of age, the AL range between the 3rd and 95 th percentile was $2.07 \mathrm{~mm}$ and $1.92 \mathrm{~mm}$ for males and females, respectively, whereas at 18 years of age, the range was $4.82 \mathrm{~mm}$ and $4.47 \mathrm{~mm}$ for males and females, respectively.

Both $\mathrm{AL}$ and $\mathrm{AL} / \mathrm{CR}$ increased with age across all percentiles; a year-to-year change was observed for all percentiles except for percentiles $<10$ which plateaued from 15 years of age with little variation thereafter $(<0.1 \mathrm{~mm}$ for $\mathrm{AL}$ and $\leq 0.01$ for $\mathrm{AL} / \mathrm{CR})$. The year-to-year change was greater at the higher compared with the lower percentiles; for example, at the 25th percentile, the year-to-year difference in $\mathrm{AL}$ at the younger ages was approximately $0.25 \mathrm{~mm}$ compared with approximately $0.4 \mathrm{~mm}$ at 95 th percentile.

The ICC between AL and AL/CR percentiles showed a difference with age; concordance was low at the younger ages $(0.13$, 4 years; $0.18,5$ years; $0.27,6$ years; $0.35,7$ years) but steadily improved with age to $\geq 0.70$ from 10 years and $>0.75$ from 13 years $(0.77,13$ years; $0.80,14$ years; $0.79,15$ years, 0.78 at 17 and 18 years of age) (figure 4).

Age-specific and gender-specific percentiles for AL and AL/CR were computed for each of the participants in the validation data set and used to predict each participant's $\mathrm{AL}$ and $\mathrm{AL} / \mathrm{CR}$ at the 1-year follow-up visit. Compared with actual AL, predicted AL was within $0.15,0.20,0.25$ and $0.30 \mathrm{~mm}$ for $70 \%, 79 \%, 86 \%$ and $90 \%$ of the eyes, respectively. Similarly, predicted AL/CR was within $0.02,0.025$ and 0.03 of the actual AL/CR for $65 \%$, $73 \%$, and $80 \%$ of the eyes, respectively. The ICC for predicted versus actual was 0.98 for $\mathrm{AL}$ and 0.96 for $\mathrm{AL} / \mathrm{CR}$. The ICC for predicted vs actual was 0.98 for $\mathrm{AL}$ and 0.96 for $\mathrm{AL} / \mathrm{CR}$.

\section{Using percentiles to estimate probability of myopia and SE}

Increasing age, increasing $\mathrm{AL}$ and $\mathrm{AL} / \mathrm{CR}$ was associated with a more myopic refractive error $\left(r^{2}=0.45,0.70\right.$ and 0.83 for age, $\mathrm{AL}$, and $\mathrm{AL} / \mathrm{CR}$, respectively). For all ages, logistic model indicated that $\mathrm{AL} / \mathrm{CR}$ percentiles had a higher diagnostic accuracy for myopia than AL percentiles (AUROC 0.967, 95\% CI: 0.965 to 0.969 vs $0.940,95 \%$ CI: 0.937 to 0.943 ). However, a model that considered both AL and AL/CR showed a statistically significant increase in determining myopia probability (AUROC 0.975, likelihood ratio test $\mathrm{p}<0.001$ ). In examining the data further, for ages $<10$ years, a bigger difference was observed between AL/CR and AL models (AUROC 0.937, 95\% CI: 0.931 to 0.942 vs $0.869,95 \% \mathrm{CI}$ : 0.861 to 0.877 ) than for ages $\geq 10$ years (AUROC 0.934 95\%CI: 0.928 to 0.940 vs 0.889 , 95\% CI: 0.881 to 0.897 ). The AUROC curve to predict myopia using AL percentiles was significantly lower for ages $<10$ compared with 
A

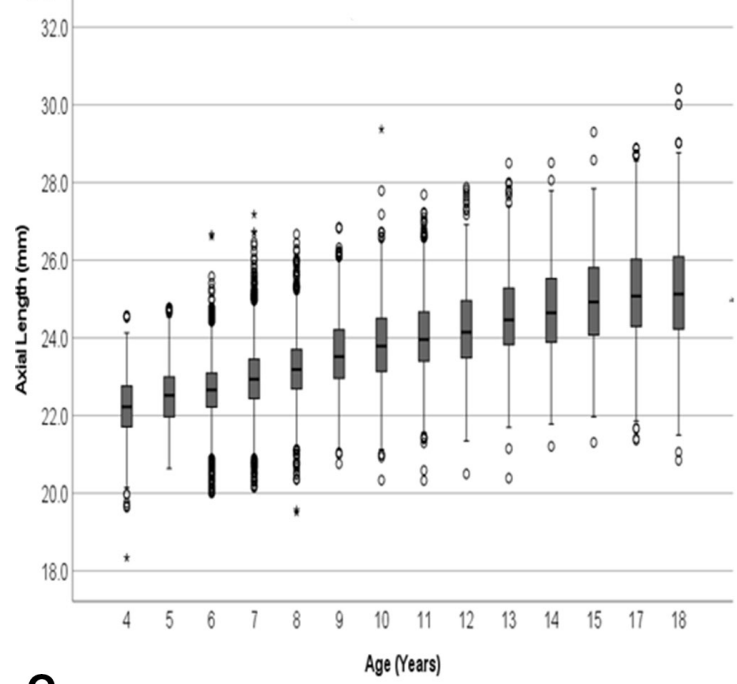

C

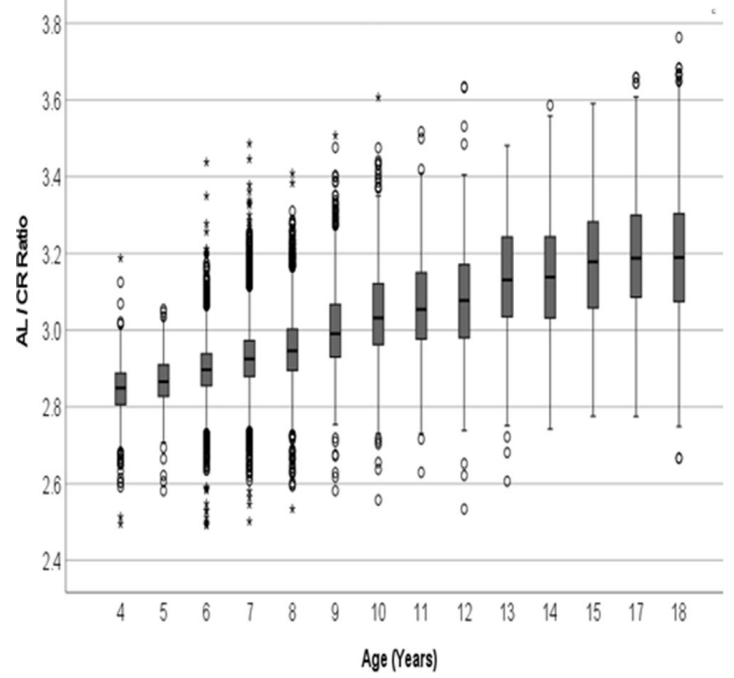

B

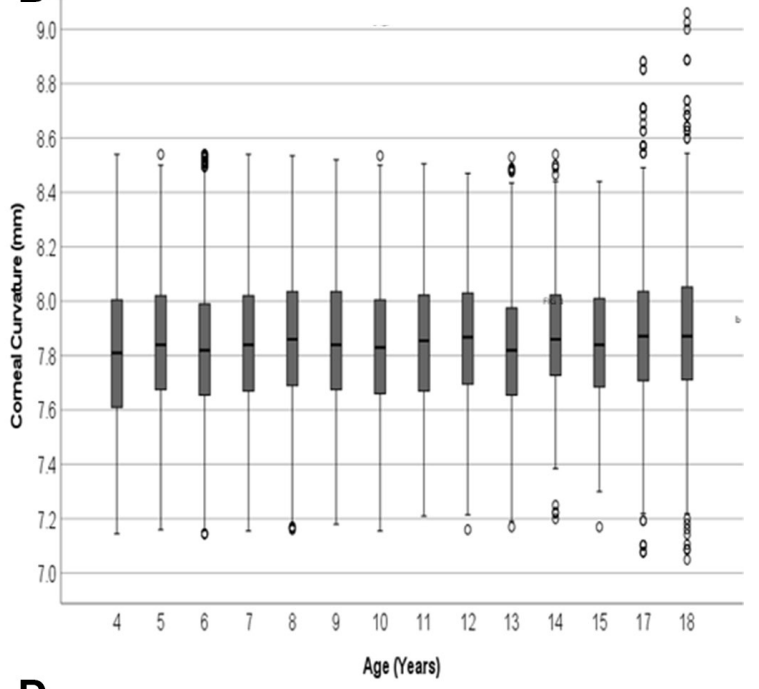

D

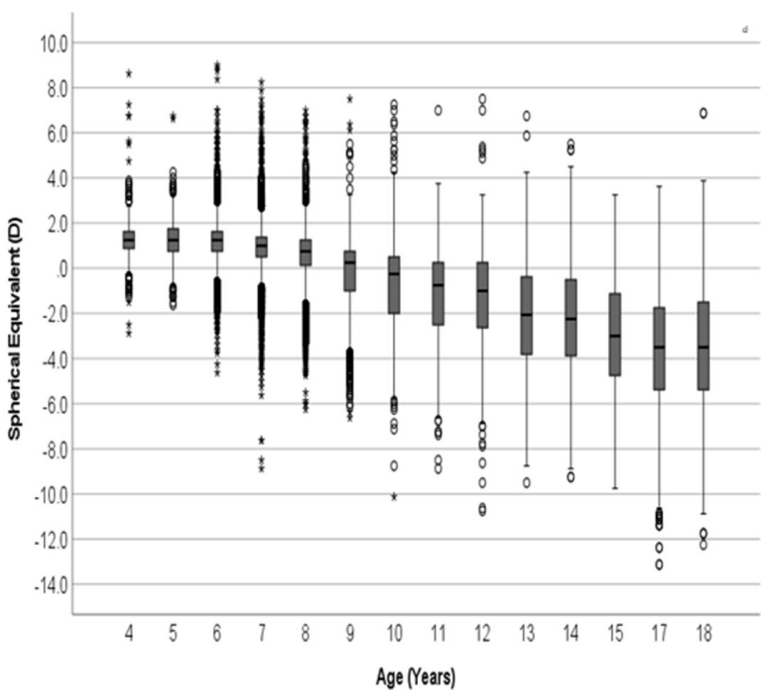

those $\geq 10$ years $(p=0.001)$ but was not different using ALCR percentiles $(p=0.581)$.

At age 10, the median AL was $23.99 \mathrm{~mm}$ and $23.51 \mathrm{~mm}$ and $\mathrm{AL} / \mathrm{CR}$ was 3.03 and 3.02 for males and females, respectively, and the mean SE was low myopia (figure 2); therefore, an increased probability that an eye may be myopic.

As seen from figure 5, at age 10 , at the 50th percentile (both $\mathrm{AL}$ and $\mathrm{AL} / \mathrm{CR}$ ) the probability of myopia prevalence was $31.8 \%$ and $37.5 \%$ whereas at the 75 th percentile the prevalence was $88.0 \%$ and $90.5 \%$ (male and female, respectively). In older children, for example, at age 12, the probability of myopia prevalence was higher even at the 50 th percentile $(74.5 \%$ and $79 \%$ for males and females, respectively).

With respect to estimating probability of myopia, the overall sensitivity and specificity of the model was $86 \%$ and $84.5 \%$, respectively (sensitivity/specificity was $84.4 / 38.9$ at 4 years; $84.2 / 95.8$ at 5 years, $84.9 / 92.1$ at 8 years, $90.2 / 82.4$ at 11 years, $96.4 / 86.5$ at 14 years and $84.0 / 86.5$ at 18 years, respectively). Specificity was high for all years excepting for 4 years old and considering only those aged 5-18 years, sensitivity and specificity was $87.4 \%$ and $88.2 \%$, respectively.
With regard to estimating $\mathrm{SE}$, the $\mathrm{R}^{2}$ of the multivariate model was 0.87 with a $\mathrm{SE}$ of the regression estimate of $0.91 \mathrm{D}$, indicating that the $95 \% \mathrm{CI}$ for any individual estimation of SE would be within $\pm 1.78 \mathrm{D}$. The accuracy of the SE model was estimated using the independent validation sample. The concordance of the estimated to the actual SE values was high $(\mathrm{ICC}=0.80)$, with estimated SE within $\pm 0.5 \mathrm{D}$ and, $\pm 1 \mathrm{D}$ of actual SE for $47 \%$ and $79 \%$ of the eyes and, within the model's limit of $\pm 1.78 \mathrm{D}$ for $96 \%$ of eyes.

\section{DISCUSSION}

The results from this cross-sectional data set of $\mathrm{AL}$ and $\mathrm{AL} / \mathrm{CR}$ for Chinese children aged 4-18 years indicate age and gender dependency. Compared with males, females have a shorter AL, a steeper corneal curvature and a slightly more myopic refractive error. The data are strikingly similar to reported data for schoolchildren from Wuhan, China. ${ }^{24}$ Comparing AL to European and other ethnic counterparts, longer AL was observed in Chinese children at all ages; the difference increased with age. ${ }^{25-29}$ For example, at 6,9 and 15 years of age, differences in AL for males from current study to those reported by Tideman et al was 
Table 3 Ocular biometry by age and gender

\begin{tabular}{|c|c|c|c|c|c|c|c|c|}
\hline \multirow[b]{2}{*}{ Age (years) } & \multicolumn{2}{|c|}{ Axial length (mm) } & \multicolumn{2}{|c|}{ Corneal curvature (mm) } & \multicolumn{2}{|c|}{$\mathrm{AL} / \mathrm{CR}$ ratio } & \multicolumn{2}{|c|}{ Spherical equivalent (D) } \\
\hline & Males & Females & Males & Females & Males & Females & Males & Females \\
\hline 4 & $22.50 \pm 0.71$ & $21.88 \pm 0.66$ & $7.88 \pm 0.27$ & $7.73 \pm 0.26$ & $2.85 \pm 0.07$ & $2.83 \pm 0.07$ & $1.14 \pm 0.88$ & $1.46 \pm 0.87$ \\
\hline 5 & $22.77 \pm 0.70$ & $22.19 \pm 0.63$ & $7.90 \pm 0.25$ & $7.78 \pm 0.25$ & $2.88 \pm 0.07$ & $2.85 \pm 0.06$ & $1.11 \pm 0.85$ & $1.38 \pm 0.76$ \\
\hline 6 & $22.95 \pm 0.66$ & $22.36 \pm 0.64$ & $7.89 \pm 0.24$ & $7.76 \pm 0.23$ & $2.91 \pm 0.07$ & $2.88 \pm 0.07$ & $1.16 \pm 0.89$ & $1.29 \pm 0.91$ \\
\hline 7 & $23.22 \pm 0.75$ & $22.66 \pm 0.68$ & $7.90 \pm 0.25$ & $7.78 \pm 0.24$ & $2.94 \pm 0.08$ & $2.91 \pm 0.08$ & $0.79 \pm 1.09$ & $0.93 \pm 1.05$ \\
\hline 8 & $23.43 \pm 0.76$ & $22.93 \pm 0.79$ & $7.90 \pm 0.25$ & $7.80 \pm 0.24$ & $2.96 \pm 0.09$ & $2.94 \pm 0.09$ & $0.53 \pm 1.29$ & $0.53 \pm 1.39$ \\
\hline 9 & $23.83 \pm 0.91$ & $23.34 \pm 0.86$ & $7.90 \pm 0.26$ & $7.80 \pm 0.24$ & $3.01 \pm 0.11$ & $2.99 \pm 0.11$ & $-0.18 \pm 1.67$ & $-0.28 \pm 1.57$ \\
\hline 10 & $24.06 \pm 1.08$ & $23.61 \pm 1.00$ & $7.88 \pm 0.23$ & $7.77 \pm 0.25$ & $3.05 \pm 0.12$ & $3.04 \pm 0.12$ & $-0.62 \pm 1.82$ & $-0.82 \pm 1.95$ \\
\hline 11 & $24.35 \pm 1.01$ & $23.77 \pm 1.00$ & $7.90 \pm 0.23$ & $7.79 \pm 0.24$ & $3.08 \pm 0.13$ & $3.05 \pm 0.12$ & $-1.16 \pm 1.93$ & $-1.26 \pm 2.06$ \\
\hline 12 & $24.38 \pm 1.08$ & $24.06 \pm 1.10$ & $7.91 \pm 0.24$ & $7.81 \pm 0.24$ & $3.08 \pm 0.14$ & $3.08 \pm 0.14$ & $-1.15 \pm 2.23$ & $-1.58 \pm 2.31$ \\
\hline 13 & $24.73 \pm 1.14$ & $24.34 \pm 1.07$ & $7.89 \pm 0.25$ & $7.75 \pm 0.21$ & $3.14 \pm 0.14$ & $3.14 \pm 0.14$ & $-1.98 \pm 2.34$ & $-2.55 \pm 2.32$ \\
\hline 14 & $25.00 \pm 1.16$ & $24.23 \pm 1.16$ & $7.93 \pm 0.23$ & $7.78 \pm 0.23$ & $3.15 \pm 0.16$ & $3.12 \pm 0.14$ & $-2.31 \pm 2.49$ & $-2.23 \pm 2.36$ \\
\hline 15 & $25.25 \pm 1.23$ & $24.72 \pm 1.19$ & $7.91 \pm 0.22$ & $7.81 \pm 0.25$ & $3.19 \pm 0.16$ & $3.17 \pm 0.14$ & $-2.88 \pm 2.58$ & $-3.12 \pm 2.49$ \\
\hline 17 & $25.41 \pm 1.34$ & $24.86 \pm 1.19$ & $7.92 \pm 0.24$ & $7.82 \pm 0.27$ & $3.21 \pm 0.16$ & $3.18 \pm 0.15$ & $-3.70 \pm 2.56$ & $-3.69 \pm 2.55$ \\
\hline 18 & $25.23 \pm 1.34$ & $24.99 \pm 1.28$ & $7.92 \pm 0.28$ & $7.83 \pm 0.27$ & $3.19 \pm 0.17$ & $3.19 \pm 0.15$ & $-3.39 \pm 2.58$ & $-3.75 \pm 2.67$ \\
\hline All ages & $23.97 \pm 1.37$ & $23.47 \pm 1.38$ & $7.90 \pm 0.25$ & $7.78 \pm 0.24$ & $3.03 \pm 0.17$ & $3.02 \pm 0.17$ & $-0.72 \pm 2.48$ & $-0.80 \pm 2.60$ \\
\hline
\end{tabular}

$A L$, axial length; $C R$, corneal radius.

$0.32 \mathrm{~mm}, 0.47 \mathrm{~mm}$ and $1.84 \mathrm{~mm}$, respectively. Methodological differences between the studies may account for some of this variation; however, it is more likely to be reflective of varying environmental influences across the different ethnic groups. Education and time outdoors have been identified as two major causal risk factors for myopia. ${ }^{5}$ In Chinese children from the Shanghai region, myopia prevalence markedly increased from 6 years of age, ${ }^{6}$ with increased educational workload cited as an important risk factor for the high prevalence. ${ }^{5}$

Interestingly, for a given AL/CR ratio; female eyes tended to have more myopia than their male counterparts with the difference manifesting especially at the onset of myopia. An AL/CR cut-off criterion of $>3.0$ still applies for detection of myopia across both genders, ${ }^{19}$ but at $\mathrm{AL} / \mathrm{CR}$ values of $\geq 3.0$, the female eye is more myopic. Although the relatively steeper corneas in females result in a more myopic refractive error, the smaller AL would in theory compensate for this myopic shift. Therefore, the more myopic error may be indicative of an AL that has elongated past the length required for emmetropia or other optical

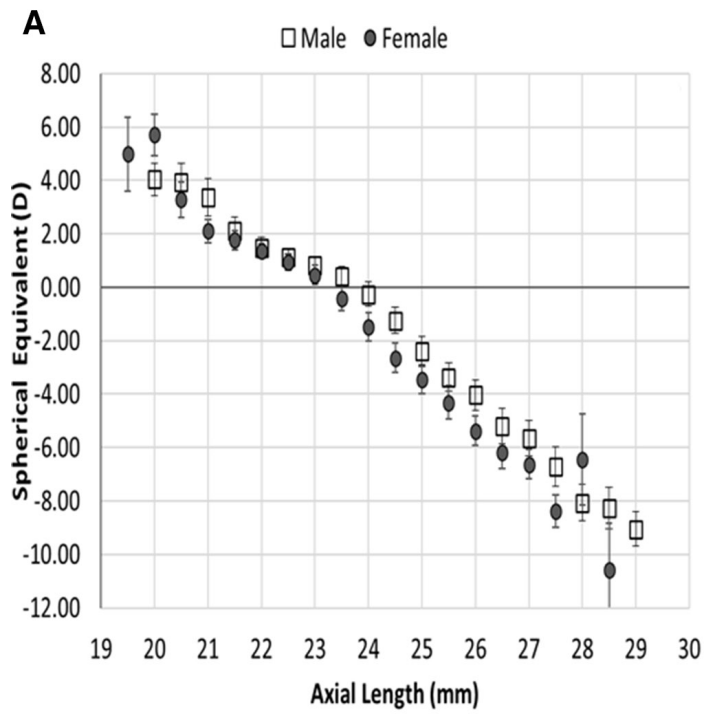

components such as the crystalline lens might be playing a role. There are reports of higher crystalline lens power in females. ${ }^{26} 27$

Previously reported AL percentiles were for children from 6 to 15 years. ${ }^{24}$ We present both $\mathrm{AL}$ and AL/CR percentiles for an extended age range from 4 to 18 years. The ICC between the $\mathrm{AL}$ and $\mathrm{AL} / \mathrm{CR}$ percentile curves was lower at the younger ages and likely reflects the greater contribution from the corneal curvature at these ages; even small changes in curvature result in significant changes in AL/CR. This also indicates that AL length alone may not be sufficient to reliably estimate eye growth and refractive state patterns at young ages.

At younger ages, that is, at 4 years, the range/spread of $\mathrm{AL}$ and AL/CR across the population was narrow but progressively expanded with age. Influence of environmental risk factors would be minimal at younger ages; the expanding range of AL and AL/ CR may suggest greater influence of environmental risk factors at older ages. Indeed, the high prevalence of myopia in many East Asian countries including China has been linked to an intensive education system with limited time outdoors. ${ }^{30}$ Furthermore,

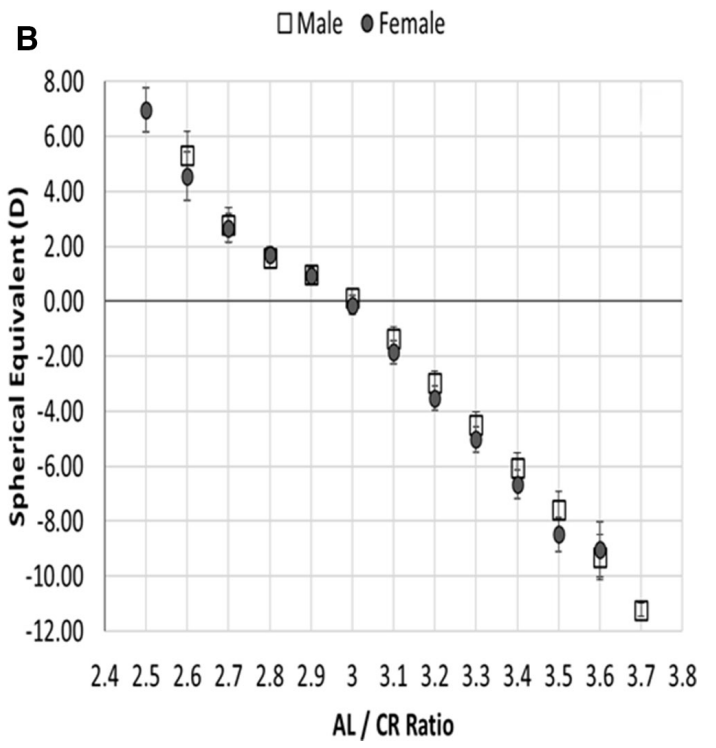


Table 4 Percentile curves for axial length

\section{Axial length percentiles}

\begin{tabular}{|c|c|c|c|c|c|c|c|c|}
\hline Age & 3 & 5 & 10 & 25 & 50 & 75 & 90 & 95 \\
\hline \multicolumn{9}{|l|}{ Males } \\
\hline 4 & 21.26 & 21.41 & 21.63 & 21.99 & 22.39 & 22.78 & 23.13 & 23.33 \\
\hline 5 & 21.49 & 21.64 & 21.87 & 22.26 & 22.69 & 23.12 & 23.51 & 23.74 \\
\hline 6 & 21.71 & 21.86 & 22.10 & 22.51 & 22.97 & 23.45 & 23.88 & 24.15 \\
\hline 7 & 21.91 & 22.07 & 22.32 & 22.75 & 23.25 & 23.76 & 24.24 & 24.54 \\
\hline 8 & 22.09 & 22.26 & 22.53 & 22.98 & 23.51 & 24.07 & 24.60 & 24.92 \\
\hline 9 & 22.27 & 22.44 & 22.72 & 23.19 & 23.76 & 24.36 & 24.93 & 25.30 \\
\hline 10 & 22.42 & 22.60 & 22.89 & 23.40 & 23.99 & 24.64 & 25.26 & 25.65 \\
\hline 11 & 22.56 & 22.75 & 23.05 & 23.58 & 24.22 & 24.90 & 25.57 & 25.99 \\
\hline 12 & 22.68 & 22.88 & 23.20 & 23.76 & 24.43 & 25.15 & 25.86 & 26.31 \\
\hline 13 & 22.78 & 22.99 & 23.33 & 23.92 & 24.62 & 25.39 & 26.14 & 26.61 \\
\hline 14 & 22.86 & 23.08 & 23.44 & 24.06 & 24.81 & 25.61 & 26.39 & 26.89 \\
\hline 15 & 22.91 & 23.15 & 23.53 & 24.19 & 24.98 & 25.82 & 26.63 & 27.14 \\
\hline 16 & 22.94 & 23.20 & 23.60 & 24.31 & 25.13 & 26.01 & 26.84 & 27.36 \\
\hline 17 & 22.95 & 23.23 & 23.66 & 24.41 & 25.28 & 26.18 & 27.04 & 27.56 \\
\hline 18 & 22.92 & 23.22 & 23.69 & 24.50 & 25.41 & 26.35 & 27.21 & 27.74 \\
\hline \multicolumn{9}{|c|}{ Females } \\
\hline 4 & 20.74 & 20.87 & 21.08 & 21.41 & 21.78 & 22.14 & 22.46 & 22.66 \\
\hline 5 & 20.96 & 21.11 & 21.33 & 21.69 & 22.10 & 22.50 & 22.87 & 23.08 \\
\hline 6 & 21.17 & 21.33 & 21.56 & 21.96 & 22.41 & 22.85 & 23.26 & 23.50 \\
\hline 7 & 21.37 & 21.53 & 21.79 & 22.22 & 22.70 & 23.19 & 23.63 & 23.90 \\
\hline 8 & 21.56 & 21.73 & 22.00 & 22.46 & 22.98 & 23.51 & 23.99 & 24.28 \\
\hline 9 & 21.73 & 21.92 & 22.21 & 22.70 & 23.25 & 23.82 & 24.34 & 24.65 \\
\hline 10 & 21.89 & 22.09 & 22.40 & 22.92 & 23.51 & 24.11 & 24.67 & 25.01 \\
\hline 11 & 22.04 & 22.25 & 22.57 & 23.12 & 23.75 & 24.39 & 24.98 & 25.34 \\
\hline 12 & 22.18 & 22.39 & 22.73 & 23.31 & 23.97 & 24.65 & 25.28 & 25.66 \\
\hline 13 & 22.29 & 22.53 & 22.88 & 23.49 & 24.19 & 24.90 & 25.55 & 25.95 \\
\hline 14 & 22.40 & 22.64 & 23.02 & 23.66 & 24.39 & 25.13 & 25.81 & 26.22 \\
\hline 15 & 22.48 & 22.74 & 23.14 & 23.81 & 24.57 & 25.34 & 26.05 & 26.47 \\
\hline 16 & 22.55 & 22.82 & 23.24 & 23.95 & 24.75 & 25.54 & 26.27 & 26.70 \\
\hline 17 & 22.59 & 22.89 & 23.33 & 24.08 & 24.91 & 25.73 & 26.46 & 26.90 \\
\hline 18 & 22.61 & 22.93 & 23.41 & 24.19 & 25.05 & 25.89 & 26.64 & 27.08 \\
\hline
\end{tabular}

the rate of change in $\mathrm{AL}$ and $\mathrm{AL} / \mathrm{CR}$ varied between the lower and higher percentiles. The overall changes from 4 to 18 years for males/females at the 25 th percentile was $2.51 / 2.78 \mathrm{~mm}$ for $\mathrm{AL}$ and $0.29 / 0.32$ for $\mathrm{AL} / \mathrm{CR}$ whereas at the 95 thpercentile, it was $4.41 / 4.59 \mathrm{~mm}$ and $0.58 / 0.60$ for $\mathrm{AL}$ and $\mathrm{AL} / \mathrm{CR}$, respectively. Probably, the higher percentiles include eyes with greater exposure to environmental risk factors or eyes with syndromic associations, pathological myopia (genetic variant), parental myopia or a combination of factors. From 15 years of age, only $<10$ th percentile plateaued $(\leq 0.1 \mathrm{~mm} \mathrm{~s}$ variation in $\mathrm{AL}$ ) whereas other percentiles continued to change. Considering other data from China, in a population from Wuhan, AL appeared to stabilise only for those in the first quartile beyond 12 years of age, ${ }^{24}$ whereas in a cohort from Guangzhou, both SE and AL continued to change from childhood to adulthood, was greater in those with parental myopia and stabilised around adulthood (20 years). ${ }^{31}$ This indicates that for most of the Chinese children, eye length is increasing even at 17 years of age. In comparison, in European children, change in AL was observed only for percentiles above $50 \%$, was at a much slower pace and the increase in AL from 5 years to adulthood only $1.28 \mathrm{~mm} / 2.5 \mathrm{~mm}$ for males/ females ${ }^{28}$ The rate of change in Chinese children from the current study was approximately $2.5 \times$ greater and has implications for risk of high myopia.
Table 5 Percentile curves for axial length (AL)/corneal radius (CR) curvature

\begin{tabular}{|c|c|c|c|c|c|c|c|c|}
\hline \multirow[b]{2}{*}{ Age } & \multicolumn{8}{|c|}{ AL/CR percentiles } \\
\hline & 3 & 5 & 10 & 25 & 50 & 75 & 90 & 95 \\
\hline \multicolumn{9}{|l|}{ Males } \\
\hline 4 & 2.74 & 2.75 & 2.77 & 2.81 & 2.84 & 2.88 & 2.91 & 2.93 \\
\hline 5 & 2.76 & 2.78 & 2.80 & 2.84 & 2.88 & 2.92 & 2.96 & 2.99 \\
\hline 6 & 2.78 & 2.80 & 2.82 & 2.86 & 2.91 & 2.96 & 3.01 & 3.04 \\
\hline 7 & 2.80 & 2.82 & 2.84 & 2.89 & 2.94 & 3.00 & 3.06 & 3.09 \\
\hline 8 & 2.82 & 2.84 & 2.87 & 2.92 & 2.97 & 3.04 & 3.10 & 3.14 \\
\hline 9 & 2.83 & 2.85 & 2.89 & 2.94 & 3.00 & 3.08 & 3.14 & 3.19 \\
\hline 10 & 2.85 & 2.87 & 2.90 & 2.96 & 3.03 & 3.11 & 3.19 & 3.23 \\
\hline 11 & 2.86 & 2.89 & 2.92 & 2.98 & 3.06 & 3.14 & 3.23 & 3.28 \\
\hline 12 & 2.87 & 2.90 & 2.94 & 3.01 & 3.09 & 3.18 & 3.26 & 3.32 \\
\hline 13 & 2.88 & 2.91 & 2.95 & 3.02 & 3.11 & 3.21 & 3.30 & 3.36 \\
\hline 14 & 2.89 & 2.92 & 2.96 & 3.04 & 3.14 & 3.24 & 3.33 & 3.40 \\
\hline 15 & 2.89 & 2.93 & 2.97 & 3.06 & 3.16 & 3.26 & 3.37 & 3.43 \\
\hline 16 & 2.90 & 2.93 & 2.98 & 3.07 & 3.18 & 3.29 & 3.39 & 3.46 \\
\hline 17 & 2.89 & 2.93 & 2.99 & 3.09 & 3.20 & 3.32 & 3.42 & 3.49 \\
\hline 18 & 2.89 & 2.93 & 2.99 & 3.10 & 3.22 & 3.34 & 3.45 & 3.51 \\
\hline \multicolumn{9}{|c|}{ Females } \\
\hline 4 & 2.71 & 2.72 & 2.74 & 2.78 & 2.81 & 2.85 & 2.88 & 2.89 \\
\hline 5 & 2.73 & 2.75 & 2.77 & 2.81 & 2.85 & 2.89 & 2.93 & 2.95 \\
\hline 6 & 2.75 & 2.77 & 2.80 & 2.84 & 2.89 & 2.94 & 2.98 & 3.01 \\
\hline 7 & 2.77 & 2.79 & 2.82 & 2.87 & 2.92 & 2.98 & 3.03 & 3.06 \\
\hline 8 & 2.79 & 2.81 & 2.84 & 2.90 & 2.96 & 3.02 & 3.08 & 3.11 \\
\hline 9 & 2.81 & 2.83 & 2.87 & 2.92 & 2.99 & 3.06 & 3.12 & 3.16 \\
\hline 10 & 2.83 & 2.85 & 2.89 & 2.95 & 3.02 & 3.10 & 3.17 & 3.21 \\
\hline 11 & 2.85 & 2.87 & 2.91 & 2.97 & 3.05 & 3.13 & 3.21 & 3.26 \\
\hline 12 & 2.86 & 2.88 & 2.92 & 2.99 & 3.08 & 3.16 & 3.25 & 3.30 \\
\hline 13 & 2.87 & 2.90 & 2.94 & 3.02 & 3.10 & 3.20 & 3.28 & 3.34 \\
\hline 14 & 2.88 & 2.91 & 2.96 & 3.03 & 3.13 & 3.22 & 3.32 & 3.37 \\
\hline 15 & 2.89 & 2.92 & 2.97 & 3.05 & 3.15 & 3.25 & 3.35 & 3.41 \\
\hline 16 & 2.90 & 2.93 & 2.98 & 3.07 & 3.17 & 3.28 & 3.37 & 3.43 \\
\hline 17 & 2.90 & 2.94 & 2.99 & 3.08 & 3.19 & 3.30 & 3.40 & 3.46 \\
\hline 18 & 2.91 & 2.95 & 3.00 & 3.10 & 3.21 & 3.32 & 3.42 & 3.49 \\
\hline
\end{tabular}

The percentiles could be used to determine the probability of myopia and therefore screen/identify those at risk of myopia. The probability of myopia increased significantly from age 10 for both genders ( $\mathrm{AL} / \mathrm{CR}>3.0$; $\mathrm{AL}>23.5 \mathrm{~mm}$ ), however, at higher percentiles there was a greater risk of myopia even at younger ages. Using AL, AL/CR and age in the model, myopia probability for 10-year-old males/females at 50th percentile was 31.8/37.5\% but increased to $>95 \%$ for both genders at 15 years of age. The sensitivity/ specificity of the model in estimating myopia probability was $84.5 / 86.0$ (4-18 years) and increased to $87.4 / 88.2$ on excluding 4 years old. Placing these results in context of screening methods employed to detect myopia, the sensitivity/ specificity of (1) non-cycloplegic refraction alone and (2) noncycloplegic refraction with uncorrected VA in detecting myopia in Chinese children aged 6-12 years from Shanghai region was estimated at 85.57/87.4 and 85.1/88.6, respectively, ${ }^{32}$ and are comparable to the sensitivity/specificity of the current model using AL, AL/CR and age. From a public health perspective, these results hold significance; $\mathrm{AL}$ and $\mathrm{AL} / \mathrm{CR}$ values obtained using modern biometers are rapid, objective, require less time and resources and are less prone to errors compared with VA or non-cycloplegic assessments (eg, VA assessment is prone to variations in the type of charts, examination site); therefore, the 

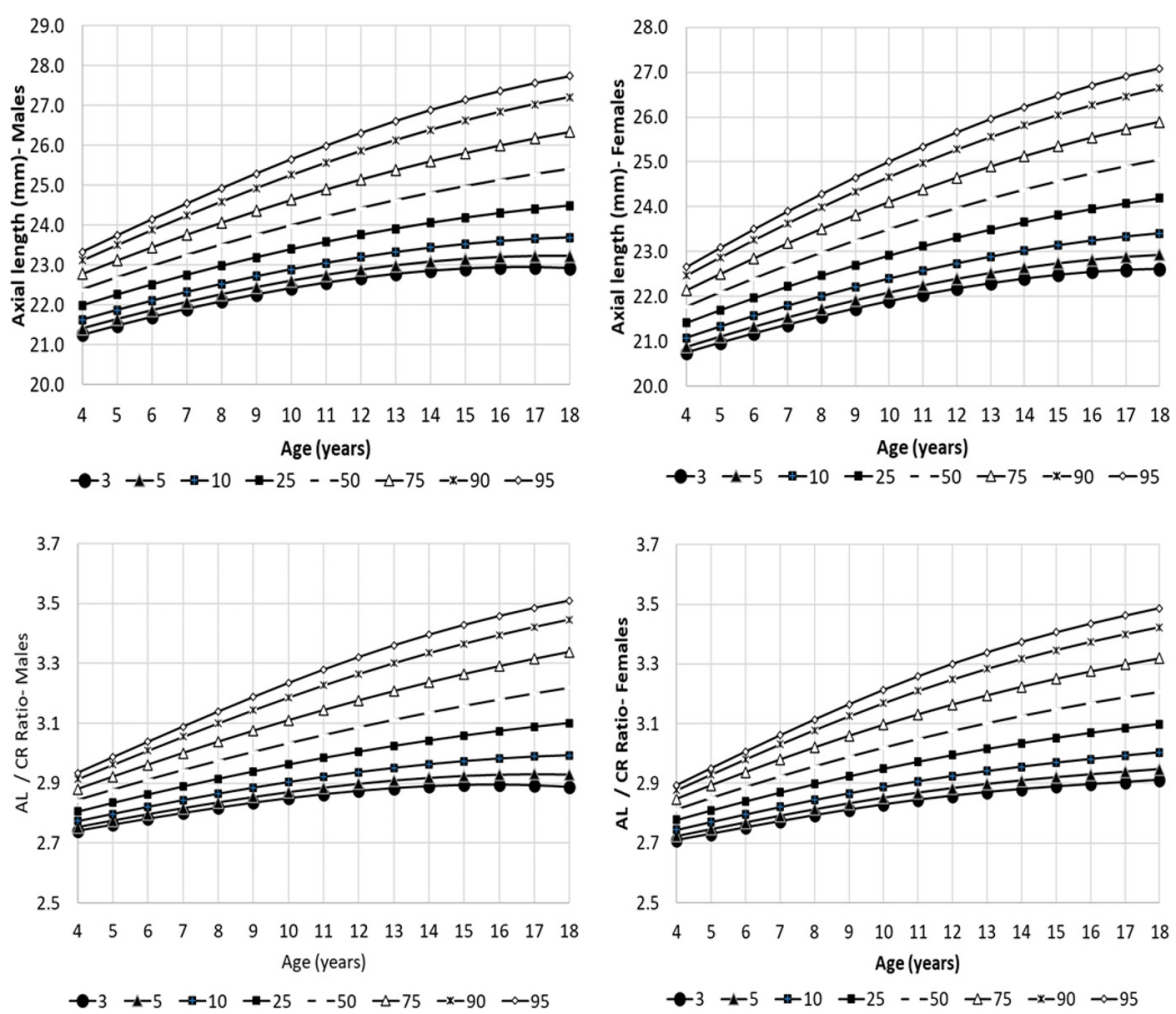

Figure 3 Age-specific and gender-specific percentile curves for axial length (AL) and AL/corneal radius (CR) curvature.

method can be utilised for large scale screening in countries with high prevalence.

In the European population, $\mathrm{AL}$ percentiles above the median were at $>50 \%$ risk of myopia in adulthood, ${ }^{28} 33$ whereas the current data indicates that Chinese children at $\geq 50$ th percentiles (for both $\mathrm{AL}$ and $\mathrm{AL} / \mathrm{CR}$ ) had a $>95 \%$ probability of myopia by age 15 . A similarly high probability of myopia prevalence $(>50 \%$ for above median AL percentiles at age 15) was reported for the study from Wuhan. ${ }^{24}$ The various cut-offs for estimating future myopia risk reinforce the need for ethnicity and region-specific percentiles; more importantly, they indicate the need for closer monitoring and appropriate interventions for those that fall at or exceed the cut-off criteria. Although the percentile charts can

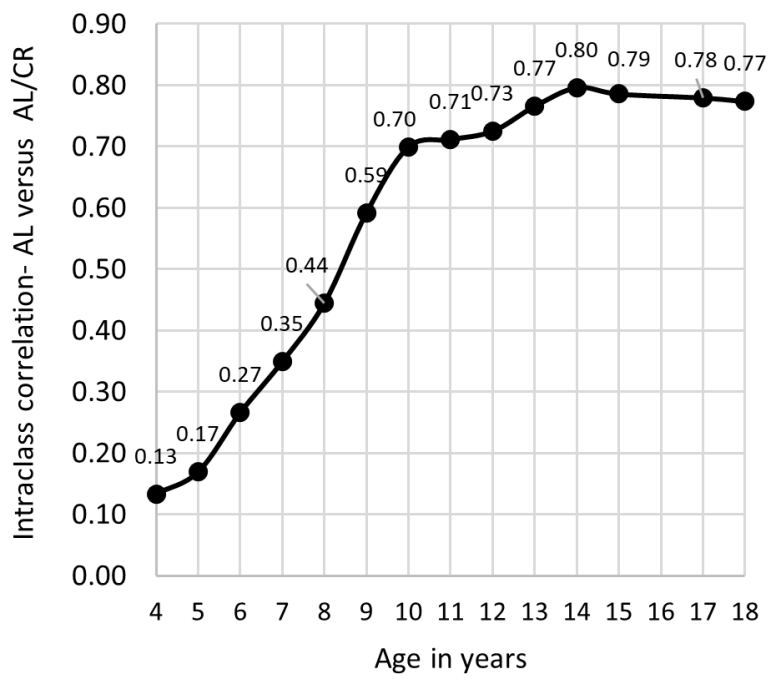

Figure 4 Intraclass correlation for axial length (AL) versus $A L /$ corneal radius (CR) curvature by age. be used to estimate myopia probability as well as monitor future changes in AL, the results suggest that it is difficult to estimate the refractive error of an individual eye with accuracy.

The large data set with a wide age range, stratification by gender and utilisation of cycloplegic autorefraction are some of the key strengths of this analysis; however, the non-homogeneity of the sample across specific ages and the lack of data for 16 years old was a limitation. Importantly, the use of percentiles rather than use of absolute values does not allow for comparison between eyes/individuals from different populations or different environments. Other limitations are the cross-sectional data with a single assessment per individual; the lack of longitudinal data is an inherent risk for projections underlying future risk of myopia. Additionally, although the ICC was high, the actual to predicted $\mathrm{AL}$ and $\mathrm{AL} / \mathrm{CR}$ varied and was $\pm 0.3 \mathrm{~mm}$ for $90 \%$ of the eyes. The reasons for variability are not entirely clear but may be related to measurement variability or the use of cross-sectional data in the development of the percentile curves. Another potential limitation of the $\mathrm{AL}$ and $\mathrm{AL} / \mathrm{CR}$ percentiles derived from urban Chinese children is that they are unable to be used across other groups that are not similar in distribution. The AL of children and their year-to-year growth pattern varies between ethnicities and requires development of percentile curves that are representative of that population. Additionally, even within a given ethnic population, variability in exposure to risk factors (eg, differences between rural vs urban areas) may influence eye growth patterns and thus limit the applicability of the percentiles. ${ }^{30}$ Finally, these percentiles are only applicable to children on simple corrective strategies for myopia.

In summary, age-specific and sex-specific $\mathrm{AL}$ and $\mathrm{AL} / \mathrm{CR}$ percentile charts are useful for monitoring changes in $\mathrm{AL}$ and $\mathrm{AL} / \mathrm{CR}$ over time and to estimate the probability of myopia. Importantly, myopia probability estimates using $\mathrm{AL}, \mathrm{AL} / \mathrm{CR}$ and age are comparable to screening techniques utilising non 

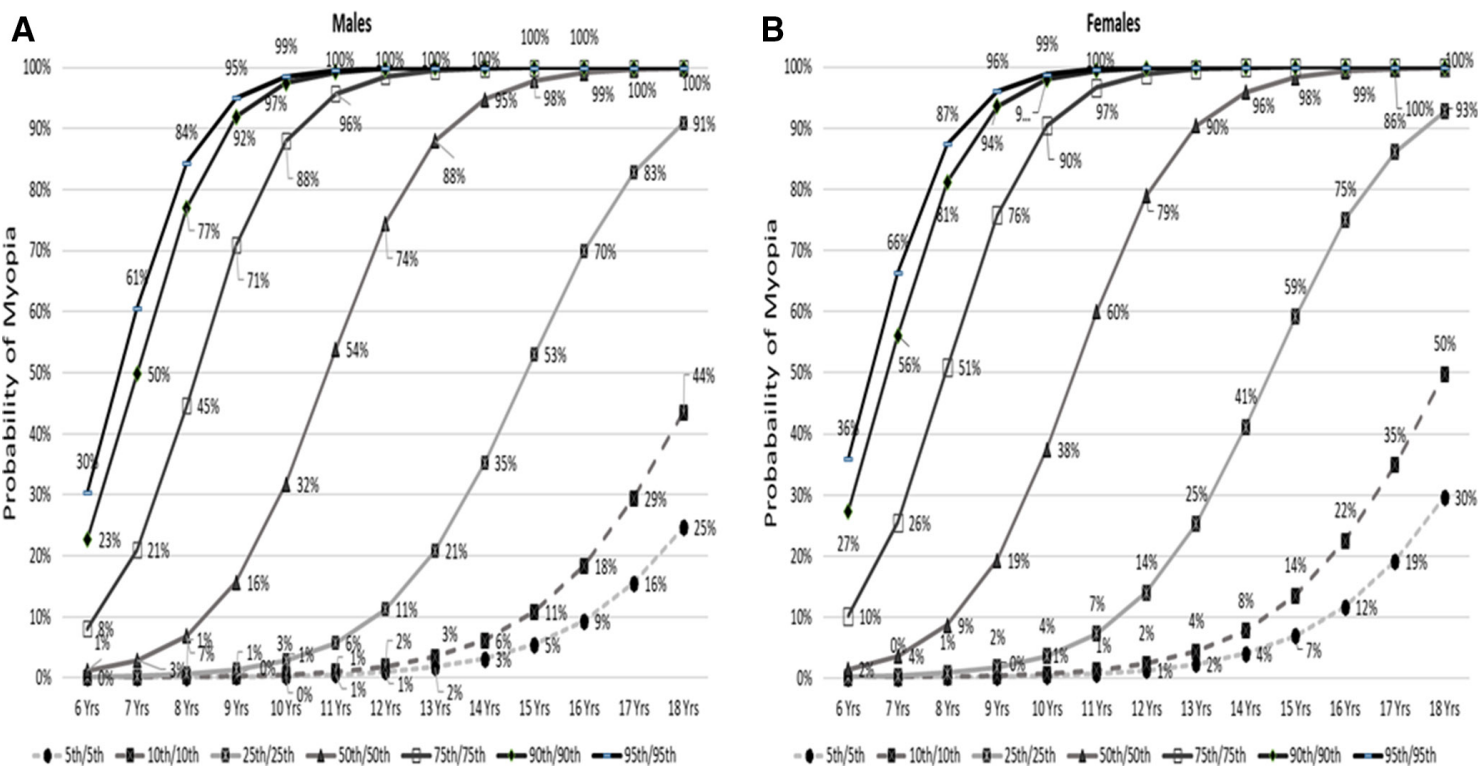

Figure 5 Probability of myopia prevalence by age and both axial length/axial length/corneal curvature percentiles in (A) males and (B) females.

cycloplegic refraction and uncorrected VA but have a higher net benefit as the biometry technique is rapid, accurate, objective and less resource intensive.

Acknowledgements The authors would like to express their sincere gratitude to the various study teams involved in the studies.

Contributors XH: conceptualisation, investigation, methodology, validation, analysis, writing, final approval; PS: conceptualisation, investigation, methodology, analysis, validation, writing, final approval; TN: conceptualisation, investigation, methodology, analysis, validation, writing, final approval; JW: investigation, methodology, writing, final approval; SX: investigation, methodology, analysis, writing, final approval; RW: methodology, writing, final approval; LD: investigation, methodology, analysis, writing, final approval; JC: investigation, methodology, analysis, writing, final approval; HZ: conceptualisation, investigation, methodology, resources, writing, final approval; XX: conceptualisation, investigation, methodology, interpretation, writing, final approval.

Funding (A) Excellent Discipline Leader Cultivation Program of Shanghai Three Year Action Plan on Strengthening Public Health System Construction (GWV-10.2-XD09) (B) 3-year Action Program of Public Health (2020-2022) (No.GWV-9.1) (C) National Key R\&D Program (No.2019YFC0840607) (D) National Science and Technology Major Project of China (No.2017ZX09304010) and (E) Brien Holden Vision Institute. The studies were supported by Shanghai Eye Disease Prevention and Treatment Center, Shanghai, China. The publication is supported in part by Oculus, Germany.

Disclaimer The funders had no role in the design or conduct of this research.

Competing interests $\mathrm{XH}$ : Employee of Shanghai Eye Disease Prevention and Treatment Center (SEDPTC); PS: Employee of Brien Holden Vision Institute (BHVI), TN: Employee of BHVI; JW: None, SX: None; RW: Employee of BHVl; LD: None, JC: None, HZ- None, XX: Employee of SEDPTC. BHVI and Oculus, Wetzlar, Germany have a commercial relation. BHVI and SEDPTC have a collaboration agreement.

Patient consent for publication Not required.

Provenance and peer review Not commissioned; externally peer reviewed.

Data availability statement Data are available on reasonable request. The data that support the findings of this study are available from Prof Xun Xu drxuxun@sjtu. edu.cn but restrictions apply to the availability of these data, which were used under licence for the current study, and so are not publicly available.

Open access This is an open access article distributed in accordance with the Creative Commons Attribution Non Commercial (CC BY-NC 4.0) license, which permits others to distribute, remix, adapt, build upon this work non-commercially, and license their derivative works on different terms, provided the original work is properly cited, appropriate credit is given, any changes made indicated, and the use is non-commercial. See: http://creativecommons.org/licenses/by-nc/4.0/.

ORCID iDs

Xiangui He http://orcid.org/0000-0002-8938-1879

Padmaja Sankaridurg http://orcid.org/0000-0001-5537-6193
Jingjing Wang http://orcid.org/0000-0003-2279-9514

Xun Xu http://orcid.org/0000-0002-4246-4343

\section{REFERENCES}

1 Holden BA, Fricke TR, Wilson DA, et al. Global prevalence of myopia and high myopia and temporal trends from 2000 through 2050. Ophthalmology 2016;123:1036-42.

2 Ang M, Flanagan JL, Wong CW, et al. Review: myopia control strategies recommendations from the $2018 \mathrm{WHO} / \mathrm{APB} / \mathrm{BHVI}$ meeting on myopia. $\mathrm{Br}$ J Ophthalmol 2020;104:bjophthalmol-2019-315575--1487.

3 Tideman JWL, Snabel MCC, Tedja MS, et al. Association of axial length with risk of Uncorrectable visual impairment for Europeans with myopia. JAMA Ophthalmol 2016;134:1355-63.

4 Wong Y-L, Sabanayagam C, Ding Y, et al. Prevalence, risk factors, and impact of myopic macular degeneration on visual impairment and functioning among adults in Singapore. Invest Ophthalmol Vis Sci 2018;59:4603-13.

5 Morgan IG, Wu P-C, Ostrin LA, et al. Imi risk factors for myopia. Invest Ophthalmol Vis Sci 2021;62:3

6 Ma Y, Qu X, Zhu X, et al. Age-Specific prevalence of visual impairment and refractive error in children aged 3-10 years in Shanghai, China. Invest Ophthalmol Vis Sci 2016;57:6188-96.

7 Lin LLK, Shih YF, Hsiao CK, et al. Prevalence of myopia in Taiwanese schoolchildren: 1983 to 2000. Ann Acad Med Singap 2004;33:27-33.

8 French AN, Morgan IG, Burlutsky G, et al. Prevalence and 5- to 6-year incidence and progression of myopia and hyperopia in Australian schoolchildren. Ophthalmology 2013;120:1482-91.

9 Jorge J, Braga A, Queirós A. Changes in myopia prevalence among first-year university students in 12 years. Optom Vis Sci 2016;93:1262-7.

10 Sankaridurg PR, Holden BA. Practical applications to modify and control the development of ametropia. Eye 2014;28:134-41.

11 Baird PN, Saw S-M, Lanca C, et al. Myopia. Nat Rev Dis Primers 2020;6:99.

12 Zadnik K, Sinnott LT, Cotter SA, et al. Prediction of juvenile-onset myopia. JAMA Ophthalmol 2015;133:683-9.

13 Sankaridurg P, He X, Naduvilath T, et al. Comparison of noncycloplegic and cycloplegic autorefraction in categorizing refractive error data in children. Acta Ophthalmol 2017:95:e633-40

14 Xiong $\mathrm{S}$, Lv M, Zou H, et al. Comparison of refractive measures of three Autorefractors in children and adolescents. Optom Vis Sci 2017;94:894-902.

15 Pesudovs K, Weisinger HS. A comparison of autorefractor performance. Optom Vis Sci 2004;81:554-8.

16 Rosenfield M, Chiu NN. Repeatability of subjective and objective refraction. Optom Vis Sci 1995;72:557-79.

17 Vogel A, Dick HB, Krummenauer F. Reproducibility of optical biometry using partial coherence interferometry : intraobserver and interobserver reliability. J Cataract Refract Surg 2001;27:1961-8.

18 Song JS, Yoon DY, Hyon JY, et al. Comparison of ocular biometry and refractive outcomes using IOL master 500, IOL master 700, and Lenstar LS900. Korean J Ophthalmol 2020;34:126-32. 
19 He X, Zou H, Lu L, et al. Axial length/corneal radius ratio: association with refractive state and role on myopia detection combined with visual acuity in Chinese schoolchildren. PLOS One 2015;10:e0111766.

20 Grosvenor T, Scott R. Role of the axial length/corneal radius ratio in determining the refractive state of the eye. Optom Vis Sci 1994;71:573-9.

21 He X, Sankaridurg P, Xiong S, et al. Shanghai time outside to reduce myopia trial: design and baseline data. Clin Exp Ophthalmol 2019;47:171-8.

22 He X, Zhao R, Sankaridurg P, et al. Design and methodology of the Shanghai child and adolescent large-scale eye study (scale). Clin Exp Ophthalmol 2018:46:329-38.

23 Cole TJ, Green PJ. Smoothing reference centile curves: the LMS method and penalized likelihood. Stat Med 1992;11:1305-19.

24 Sanz Diez P, Yang L-H, Lu M-X, et al. Growth curves of myopia-related parameters to clinically monitor the refractive development in Chinese schoolchildren. Graefes Arch Clin Exp Ophthalmol 2019;257:1045-53.

25 Zadnik K, Manny RE, Yu JA, et al. Ocular component data in schoolchildren as a function of age and gender. Optom Vis Sci 2003;80:226-36.

26 Twelker JD, Mitchell GL, Messer DH, et al. Children's ocular components and age, gender, and ethnicity. Optom Vis Sci 2009;86:918-35.
27 Momeni-Moghaddam H, Hashemi H, Zarei-Ghanavati S, et al. Four-Year change in ocular biometric components and refraction in schoolchildren: a cohort study. J Curr Ophthalmol 2019;31:206-13.

28 Tideman JWL, Polling JR, Vingerling JR, et al. Axial length growth and the risk of developing myopia in European children. Acta Ophthalmol 2018;96:301-9.

29 Rauscher FG, Francke M, Hiemisch A, et al. Ocular biometry in children and adolescents from 4 to 17 years: a cross-sectional study in central Germany. Ophthalmic Physiol Opt 2021;41:496-511.

30 Morgan IG, French AN, Ashby RS, et al. The epidemics of myopia: aetiology and prevention. Prog Retin Eye Res 2018;62:134-49.

31 Liao C, Ding X, Han X, et al. Role of parental refractive status in myopia progression: 12-year annual observation from the Guangzhou twin eye study. Invest Ophthalmol Vis Sci 2019;60:3499-506.

32 Lin $\mathrm{S}, \mathrm{Ma} \mathrm{Y}, \mathrm{He} \mathrm{X}$, et al. Using decision curve analysis to evaluate common strategies for myopia screening in school-aged children. Ophthalmic Epidemiol 2019;26:286-94.

33 Truckenbrod C, Meigen C, Brandt M, et al. Longitudinal analysis of axial length growth in a German cohort of healthy children and adolescents. Ophthalmic Physiol Opt 2021:41:532-40. 